\title{
Ocular trauma: from epidemiology to war-related injuries
}

\author{
Ferenc Kuhn
}

Received: 23 September 2011 / Accepted: 26 September 2011 /Published online: 14 October 2011

(C) Springer-Verlag 2011

In the current issue of Graefe's, the reader finds numerous excellent articles dealing with ocular traumatology.

Shah et al. present a large series of cases with traumatic cataract. The strength of this paper is due to the size of the cohort, the use of the standardized language of eye-injury terms (Birmingham Eye Trauma Terminology system, BETT), the prospective nature of data collection, and the inclusion of follow-up data, albeit with a relatively short duration.

Traumatic cataract is a somewhat neglected injury type. This is probably due to the fact that it is rarely the lens that determines the outcome of the case - notable exceptions include children of amblyopic age if the extraction and proper orthoptic therapy are delayed, and surgical errors such as creating a retinal break by inappropriately engaging vitreous during lens removal [1]. The reason why the prognosis of injured eyes with traumatic cataract was found to be actually worse in the database of the American Society of Ocular Trauma compared to eyes without traumatic cataract is the severity of accompanying tissue pathologies; posterior segment lesions are present in almost half of eyes with a traumatic cataract [1]. The findings of Shah et al. confirm the need to intervene rather early if lens opacity is present, but they surprisingly found worse outcome in eyes with closed-globe trauma. Unfortunately, they did not discuss this apparent paradox in their article in more detail; nevertheless, these data confirm the need for ophthalmologists not to defer surgical intervention in eyes with contusion.

F. Kuhn $(\bowtie)$

1201 11th Avenue South, Suite 300,

Birmingham, AL 35205, USA

e-mail: fkuhn@mindspring.com
The article by Rüfer et al. discusses another, even more neglected issue: the role and consequences of alcohol consumption as it relates to eye injuries. Their findings confirm the common-sense assumption that ocular trauma sustained while intoxicated is in general more severe and often caused by others (assault); it is more likely to occur on the street and at night. Crucially, ruptures were found to have a statistically significantly higher incidence in the intoxicated group, probably reflecting the coordination difficulties which alcohol causes. This exemplary study finally provides exact data concerning the influence of alcohol on eye injury occurrence; furthermore, it also reveals useful information about the management of the injured eyes. The author of this editorial is especially pleased to see that Rüfer et al. decided in favor of performing primary pars plana vitrectomy in a substantial proportion of their cases; such early vitrectomy offers the potential of preventing complications that otherwise might have occurred. Another noteworthy finding in their study was the results seen when using the Ocular Trauma Score (OTS): although the initial visual acuities did not differ significantly between the two groups, the OTS raw numbers did, predicting a worse outcome in the intoxicated group.

The study by Jovanovic et al. shows the severity of eye injuries incurred by those not wearing a seatbelt. No fewer than $26 \%$ of the individuals suffered bilateral eye injuries, and almost $30 \%$ of eyes became blind (no light perception). Reading in the Results section that half of those injured were under the influence of alcohol, and that almost half of those injured were the driver of the car, one has to wonder whether alcohol also played a role in causing the crash. This suspicion is confirmed in the Discussion section, and again points to the importance of the work by Rüfer et al. The authors do not supply treatment data, leaving the 
readers to wonder whether early intervention may have saved some eyes.

The report by Barak et al. on the injuries sustained by the Israeli side during the Second Lebanon War is a very sobering one, with important implications for all ophthalmologists, regardless of whether they expect to see such conflicts in their own clinical practice (to paraphrase Leonid Trotsky: You may not be interested in war or terrorism, but they are interested in you). One of the observations the authors makes relates to the low rate of utilizing protective eyewear among the soldiers, which has devastating consequences. One must wonder, in light of data revealed by the US military [2] about the increasing efficiency of the so-called improvised explosive devices, whether such protective eyewear is indeed going to offer only a reduced safety margin in the future (or, rather, the present...). Conversely, the incredible efforts in Israel to reduce the injury risks posed by incoming rockets to the civilian population by mandating the availability of a bomb-proof zone in every dwelling have proven very effective; hopefully, improved war technology will not make these efforts less fruitful. The ophthalmologist's role in this scenario is to treat the injured as best as possible, and to provide feedback for those who make policy decisions.

Another epidemiological study in this issue of Graefe's is by Soong et al., from an area of the world where such studies are less commonly performed than in Europe or the US. The study is especially valuable since it is standardized (BETT, data-collection sheets), prospective, and a large number of cases is involved, demonstrat- ing that in Malaysia the shift from workplace to home as the most common place of injury has not yet fully occurred. Another important datum is the still-high percentage of injuries caused by motor vehicle crashes. Similarly to the report from Serbia, non-wearing of the seatbelt is a crucial factor, and, just as with injuries occurring at the workplace, a relatively simple route to prevention is available. Finally, it appears from the study that non-Malaysians suffer a disproportionately higher rate of injuries; this may be the topic of a future study, analyzing whether this is due to the nature of work they do or some cultural factors.

Gaultieri et al. report a very rare event, loss of the iris in the context of globe rupture while sparing the lens - which is usually also extruded or at least dislocated. The most important information in their case, however, was the fact that the visual acuity, no light perception on presentation, improved to almost full in a month. This dramatic improvement is further evidence that eyes losing light perception due to trauma should not be abandoned, much less enucleated.

Conflict of Interest I have no conflict of interest.

\section{References}

1. Kuhn F (2010) Traumatic cataract: what, when, how. Graefes Arch Clin Exp Ophthalmol 248:1221-1223

2. Mader TH, Carroll RD, Slade CS, George RK, Ritchey JP, Neville SP (2006) Ocular war injuries of the Iraqi insurgency, JanuarySeptember 2004. Ophthalmology 113:97-104 